\title{
Investigating Mechanisms of Spatiotemporal Epidemic Spread Using Stochastic Models
}

\author{
G. J. Gibson
}

Biomathematics and Statistics Scotland, James Clerk Maxwell Building, University of Edinburgh, Mayfield Road, Edinburgh, Scotland EH9 3JZ. Accepted for publication 22 October 1996.

To enable efficient control of a virus disease in an agricultural crop, it is desirable to understand the mechanisms that underlie its spread. Observations from small-scale field experiments, in which disease incidence is intensively mapped in space and at several time points, may shed some light on these mechanisms, and a range of analytic tools is available for their analysis and interpretation. For example, spatiotemporal distance class analysis $(12,22)$ has been used to study the spatial distribution of newly detected infections in a population in relation to previous infections. Techniques based on spatiotemporal autocorrelation (1) such as STARIMA (spatiotemporal autoregressive integrated moving average) methods $(13,23,24)$ have also been applied to model changes in disease incidence over time. A related approach is the use of ordinal regression $(2,19,29)$, in which the probability of a change in the disease status of an individual at an observation time is modeled as a function of its status and the status of individuals in some neighborhood at a previous time. In common with STARIMA methods, this latter approach involves the fitting of a discretetime Markov model to the evolution of the epidemic.

For discrete-time models to be applicable, frequent observation of a process may be required. If, however, the interval between observations is long in comparison with the time scales over which the disease spreads, then a continuous-time stochastic model may be more appropriate. Although, continuous-time stochastic models have been studied in the literature for many years $(5,6,20,21)$, they have seen comparatively little use in the analysis of experimental data, with most emphasis placed on understanding their theoretical behavior and the macroscopic properties of the patterns they produce. However, recent developments in statistical methodology and computing power have led to a greater role for these models as analytic tools. Recently, Gibson and Austin (11) fitted continuous-time, spatiotemporal stochastic models to observations of a virus disease using stochastic integration methods. Their methods have been further refined using Markov chain Monte Carlo (MCMC) $(3,9,14,15,28)$ by Gibson (10).

The present paper illustrates and extends the techniques of Gibson (10) by applying them to observations of epidemics of citrus tristeza virus (CTV) in two experimental plots, each consisting of 216 trees of Washington navel orange (Citrus sinensis (L.) Osbeck) on Troyer citrange (Poncirus trifoliata (L.) Raf.), observed in Eastern Spain, where disease is spread by the vector Aphis gossypii. These data, illustrated in Figure 1, describe the distribution of CTV in the plots (which were originally disease free) as observed at three times, each 1 year apart. They were first reported and analyzed (along with several other CTV epidemics) by Gottwald et al. (12) using several methods mentioned above. The main goals of the analysis were to determine the relationship

Corresponding author: G. J. Gibson; E-mail address: gavin@bioss.sari.ac.uk

Publication no. P-1997-0114-010

(C) 1997 The American Phytopathological Society of diseased trees with each other and to detect directionality in the spread. Conclusions of Gottwald et al. (12) were that there was little evidence of anisotropy or aggregation in the patterns and that spread did not appear to occur preferentially to trees adjacent to those already diseased. The simultaneous presence of inter- and intraplot spread was suggested as the mechanism underlying the observations. The purpose of the new analysis presented here is to shed further light on the mechanisms that might underlie these data, by relating the disease maps directly to spatiotemporal stochastic models of spread. I believe the approach illustrated is a valuable addition to the methodology applicable to spatiotemporal data, which complements existing methods by allowing investigation of alternative transmission mechanisms using data in a direct, efficient manner.

Spatiotemporal stochastic models. I consider a simple stochastic model for the spread of a virus disease through a discrete population. The model is more complex than related models considered by Gibson and Gibson and Austin $(10,11)$ in that it represents virus transmission both from individuals in the population and from sources outside the population. The members of the population are represented by the vertices of a rectangular lattice. For ease of notation, I shall use $x$ to denote both the spatial coordinates of a vertex and the individual located there. An individual $x$ may be in one of two states: susceptible or diseased. I denote the set of diseased individuals at time $t$ by $D(t)$. The model considered is stochastic, meaning it represents the element of chance, or probability, in the acquisition of the disease by any susceptible individual. The probabilistic nature of disease transmission is incorporated as follows. If the individual $x$ is susceptible at time $t$ and, if $\mathrm{d} t$ is small, then $x$ acquires the disease in the interval $(t, t+$ $\mathrm{d} t$ ) with some probability that is proportional to $\mathrm{d} t$ and is related to the locations of the diseased individuals in the population. Explicitly, the probability that $x$ becomes diseased during $(t, t+\mathrm{d} t)$ is equal to $r_{a}(x) \mathrm{d} t$, in which

$$
r_{a}(x)=a_{1}+\sum_{y \in D(t)}|y-x|^{-2 a_{2}}
$$

and $|y-x|$ measures the distance between $y$ and $x$ on the lattice. The term $r_{a}(x)$ represents the rate at which the individual $x$ is acquiring the disease at time $t$, and the model is specified by the parameter vector $a=\left(a_{1}, a_{2}\right)$, in which $a_{1}, a_{2} \geq 0$. The parameter $a_{1}$ represents the rate at which any susceptible in the lattice acquires the disease due to primary infection from sources outside the lattice. Local transmission (i.e., acquisition of disease from diseased individuals in the lattice) of the disease is represented by the summation term appearing in equation 1 . It is assumed that $x$ acquires the disease from a diseased individual, $y$, with rate $|y-x|^{-2 a_{2}}$. Hence, the net rate at which $x$ acquires the disease from diseased individuals in the lattice is obtained by summing this quantity over all $y \in D(t)$. The parameter $a_{2}$ determines how the rate of local transmission decreases with the distance between $x$ and $y$. 
In this paper, equal spacing between the rows and columns of the lattice is assumed. Furthermore, distances are normalized so that this distance is unity, and || is Euclidean distance on the normalized lattice. For the CTV epidemics of Figure 1, the geometry of the orchards is somewhat more complicated than that of the square lattice just described. Nevertheless, it can be argued that, for the case of a virus disease spread by aphids, adjacency in the lattice is more important than absolute distance when considering the likelihood of transmission from a diseased tree to a susceptible tree by aphid movement. Hence, the above metric is used in preference to absolute distance.

In defining $r_{a}(x)$, as in equation 1 , I appear to ignore additional factors such as vector numbers and temperature, which undoubtedly influence the rate of disease spread. These combined effects could be modeled by a time-varying, but spatially invariant, factor that multiplies the rate $r_{a}(x)$. The precise nature of this factor, while it would affect the statistical distribution of the absolute time at which any individual acquires the disease during a run of the model, would not affect the statistical distribution of the order in which the infections occur, this being dependent only on the initial spatial distribution of disease and the parameters $a_{1}$ and $a_{2}$. Since the techniques described make use of only information on the order of occurrence of infections, and not the exact times of occurrence, then consideration of a model in which no such factor appears is justified. A rigorous justification for this simplification is given by Gibson and Austin (11).

I assume that the infective challenge decreases as a power of the distance between susceptible and diseased individuals. Other relationships clearly can be used, such as a challenge that decays exponentially with separation. Shaw $(26,27)$ has investigated the qualitative differences between patterns produced by stochastic models and alternative functional forms for local interactions, demonstrating that infective challenges that are not exponentially bounded produce patterns that mimic those observed in real epidemics. Gibson and Austin (11) demonstrated that a power-law decay better represented an epidemic of CTV reported in Marcus et al. (18) than did an exponentially bounded model, and these observations further motivate the choice of model.

The model defined by equation 1 is closely related to other models of spatial spread (e.g., contact distribution models [20, 21]). Although simple, it provides a framework for investigating hypotheses regarding the relative importance of primary and secondary transmission. Dependent on the values of the parameters $\left(a_{1}, a_{2}\right)$, equation 1 specifies very different mechanisms of spread. If $a_{2}=0$, then any diseased tree affects all susceptibles equally, regardless of relative position. At any time, therefore, all susceptibles are equally likely candidates for the next infection and the set $D(t)$ evolves as a random pattern. Large values of $a_{2}$ correspond to a local infective challenge that declines rapidly with distance, so that local transmissions are predominantly to nearest neighbors. As $a_{1} \rightarrow \infty$, the background infection rate increasingly dominates the effect of any local interactions, for any value of $a_{2}$, and the spatial distributions of disease produced by the model again assume the appearance of random patterns. If $a_{1}$ is small and $a_{2}$ is large, so that transmission is largely a nearest-neighbor interaction, it is likely that new entries into $D(t)$ will lie adjacent to individuals already in $D(t)$, placing constraints on the likely orders with which infections may occur. Figure 2 depicts the rela-

A
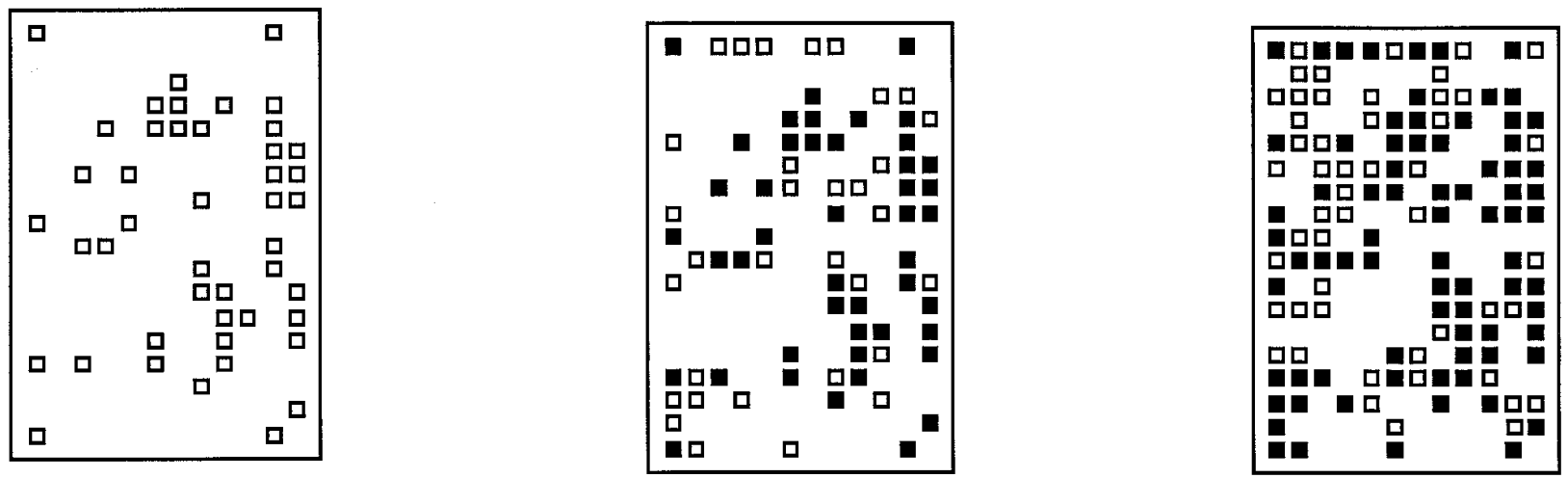

B
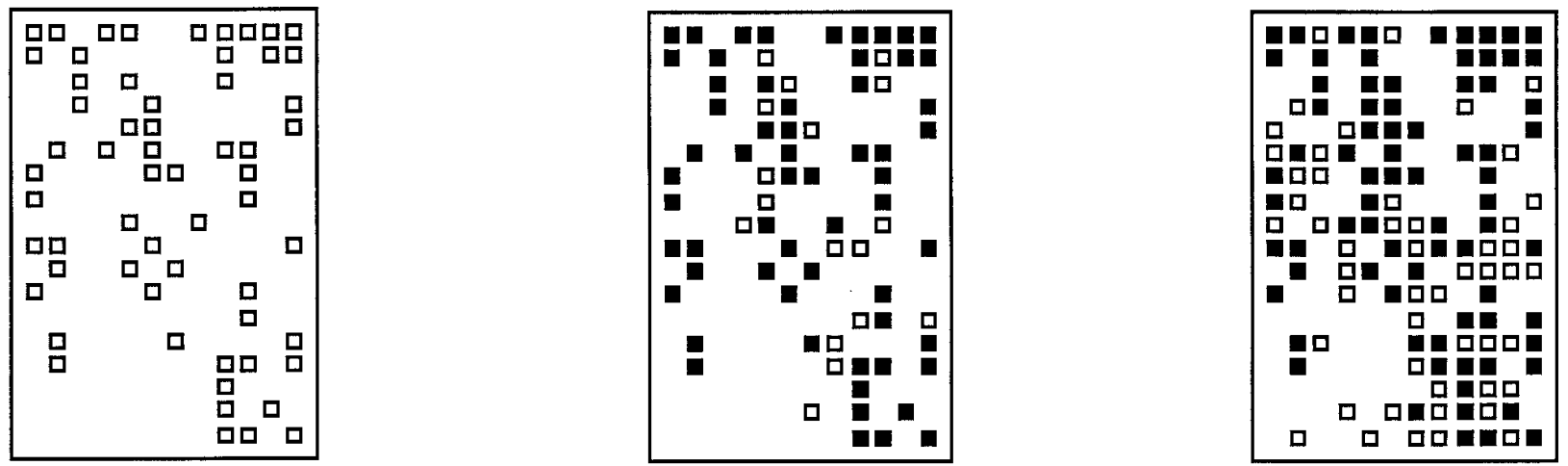

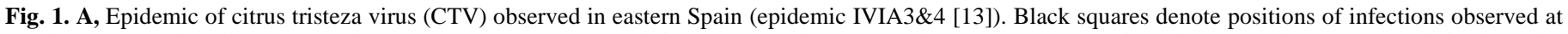

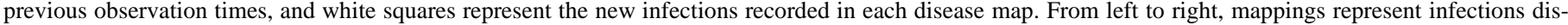

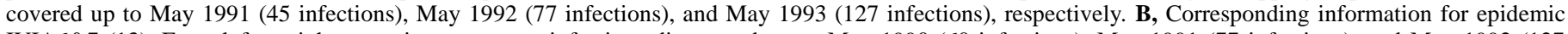

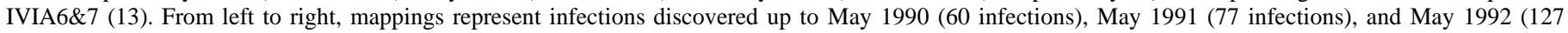
infections), respectively. 
tionship between the model parameters and the characteristics of the infection process in the model.

Model fitting with MCMC. Like that presented by Gibson and Austin (11), the model-fitting method is likelihood-based. However, here I make use of a superior computational approach described in detail and illustrated using simulated data sets and historical data in Gibson (10). The essential difficulty the method is designed to overcome is that of incomplete data. I have proposed a model for a system and wish to estimate model parameters, and the uncertainty in the estimates, from observations of the system. If information in the observations were 'complete,' in some sense, parameter estimation would be straightforward. However, some important information is not observed and the problem of parameter estimation in the absence of this information must be tackled. This class of problem is one to which MCMC methods $(3,9,14$, 15,28 ) have been successfully applied in the past and, as I show, they are highly applicable to the specific problems of this paper. In essence, MCMC methods allow the joint uncertainty in model parameters and missing data to be explored through the evolution of a Markov chain. Parameter estimation is then achieved by considering the marginal statistical properties of the model parameters.

I now describe how the problem of fitting the model defined by equation 1 to observations consisting of disease maps at discrete times can be formulated and solved in the above framework. First, consider the 'ideal' situation in which the lattice is intensively mapped at time $t_{0}$, when the set of diseased individuals is $D\left(t_{0}\right)$, and then continuously observed until time $t_{1}$. This would yield knowledge of the locations $\left(x_{1}, \ldots, x_{N}\right)$ of all new infections occurring in the interval $\left(t_{0}, t_{1}\right)$, and also the precise temporal order, $\omega$, in which they occurred. As was shown by Gibson and Austin (11), a likelihood, $G(a)=P(\omega \mid a)$, could then be evaluated for a range of parameter vectors and the values of $G(a)$ used to compare the fit of different values of $a$. However, this scenario is unlikely to arise in practical experimental systems. More commonly, and as considered here, the lattice might be mapped intensively at $t_{0}$ and $t_{1}$, but not observed during the intervening period. This would produce an observation, $E$, consisting of the initial diseased set, $D\left(t_{0}\right)$; the locations of new infections, $\left(x_{1}, \ldots, x_{N}\right)$; but not the order, $\omega$, in which these infections had occurred. It is the order, $\omega$, that corresponds to the 'missing data' discussed in the previous paragraph. Evaluate of the likelihood $\mathrm{G}(\mathrm{a})=\mathrm{P}(\mathrm{E} \mid \mathrm{a})$ requires summation (or 'integration') of the quantity $P(\omega \mid a)$ over the set of all possible orderings, $\omega$-a task that is impractical unless $N$ is small (approximately less than 12). Nevertheless, as I now describe, it is possible to estimate the relative values of $G(a)$ over the parameter range using MCMC methods, thereby, enabling estimation of model parameters.

Under the assumption that all values $a$ are a priori equiprobable, $G(a)$ is proportional to the posterior density $\pi(a \mid E)$. Although direct calculation of $\pi(a \mid E)$ is not feasible, for any ordering, $\omega$, the density $\pi(a \mid \omega)$ can be calculated. This is exploited in the MCMC approach by using a Markov chain to generate a sequence of parameter/ordering pairs $\left\{\left(a_{i}, \omega_{i}\right) \mid 1 \leq i \leq K\right\}$, in which $K$ is the number of samples generated from $\pi(a, \omega \mid E)$, the joint posterior density of parameters/orderings. An estimate of the posterior parameter density $\pi(a \mid E)$ can then be calculated as

$$
\pi(a \mid E)=\frac{1}{K} \sum_{i=1}^{K} \pi\left(a \mid \omega_{i}, E\right)
$$

The precise construction of the chain is described by Gibson (10). In summary, a new pair $\left(a_{i+1}, \omega_{i+1}\right)$ is obtained from the current pair $\left(a_{i}, \omega_{i}\right)$ by first drawing $a_{i+1}$ from the distribution $\pi\left(a \mid \omega_{i}\right)$ (which can be computed easily as in Gibson [10]). To obtain $\omega_{i+1}$, the ordering $\omega_{i}$ is 'mutated' many times by repeatedly considering pairs of temporally adjacent infections $\left(x, x^{\prime}\right)$ and stochastically reversing their order. The probability of any pair being reversed is derived from the relative likelihood of its two possible orderings, conditional on the new model parameter $a_{i+1}$. In the implementation used, the pair $\left(x, x^{\prime}\right)$ is not selected randomly. Rather, pairs of infections are examined systematically in a cyclic process that ensures that every infection (except initial and final) is considered twice per cycle. In the computations presented in this paper, $\omega_{i+1}$ is obtained from $\omega_{i}$ using 500 applications of the mutation cycle.

The generalization of the above procedure to epidemics observed at $n>2$ time points, $t_{0}, \ldots, t_{n-1}$, is as follows. A density $\pi\left(a \mid E_{j}\right)$ is calculated for $j=1, \ldots, n-1$ in which $E_{j}$ denotes observation of the sets of diseased individuals at times $t_{j-1}$ and $t_{j}$. The product of these densities is normalized to estimate the density $\pi\left(a \mid E_{1}, \ldots, E_{n-1}\right)$. If the data describe the infected set $D(t)$ at only a single time point, model fitting can be carried out by assuming the epidemic began with a single infection, and all individuals in $D(t)$ are equiprobable candidates for this infection.

Application of techniques to simulated epidemics. To illustrate the above methods in action, I first consider observations of epidemics simulated using the model defined by equation 1 with known parameter values. Figure $3 \mathrm{~A}$ to $\mathrm{D}$, respectively, depict the evolution of four simulated epidemics on an $18 \times 12$-unit lattice. The parameters selected for these scenarios are (i) $a_{1}=1.15, a_{2}=$ 3.5 (background plus short-range local interaction); (ii) $a_{1}=1.72$, $a_{2}=1.4$ (background plus local interaction); (iii) $a_{1}=0.0, a_{2}=1.0$ (purely local interaction, with initial infection randomly chosen); and (iv) $a_{1}=1.0, a_{2}=0.0$ (random process).

For these simulated epidemics, the observations comprise transitions $E_{1}, E_{2}$, and $E_{3}$, in which $E_{1}$ denotes the transition from the disease-free state to the first disease map. The number of infections occurring in the transitions $E_{1}, E_{2}$, and $E_{3}$ for these epidemics correspond to numbers in Figure 1A for simulations (i), (iii), and (iv) and Figure 1B for simulation (ii).

The likelihood $G(a)$ is typically sensitive to $a_{1}$ at low values of this parameter and insensitive at high values. For this reason, I have found it useful to apply the monotonic transformation

$$
b=\left[\log \left(1+a_{1}\right)\right]^{1 / 2}
$$

(so that $a_{1}=e^{b^{2}}-1$ ) and to carry out model fitting using the parameter space consisting of a $21 \times 21$-rectangular grid of values $\left(b, a_{2}\right)$ in which $b=0.0,0.125,0.25, \ldots, 2.5$ and $a_{2}=0.0,0.175$, $0.35, \ldots, 3.5$. The maximum value of $b$ implies that background trans-

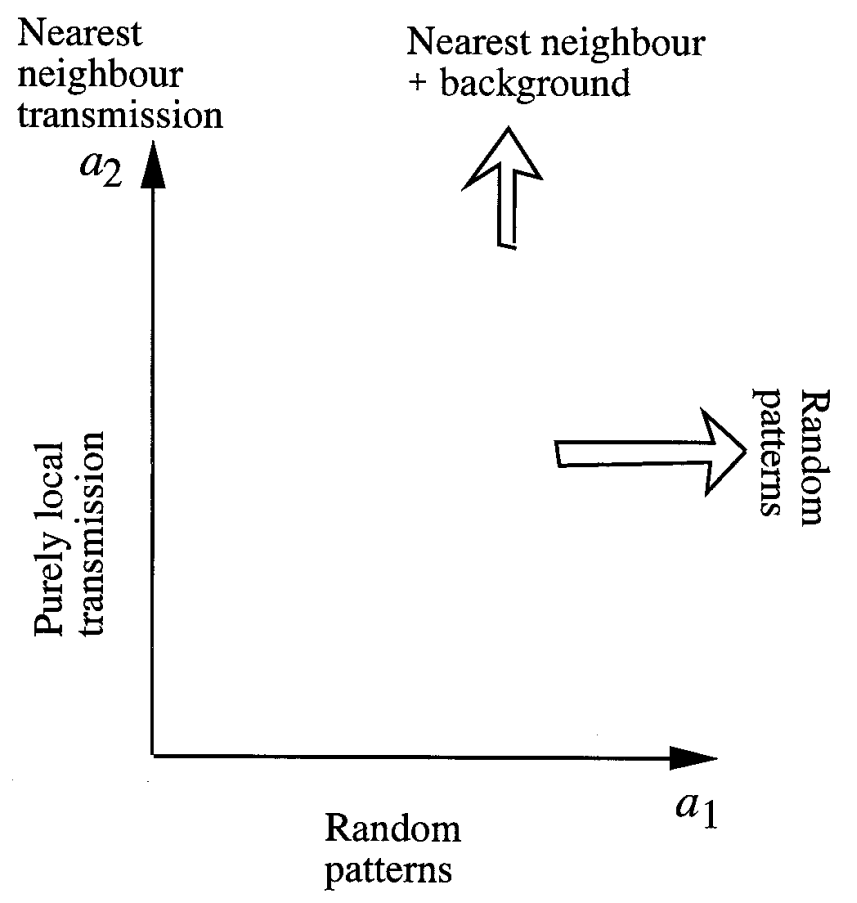

Fig. 2. Schematic representation of parameter space for model defined by equation 1 showing the correspondence between parameter values and qualitative characteristics of resulting patterns of spread. 
A

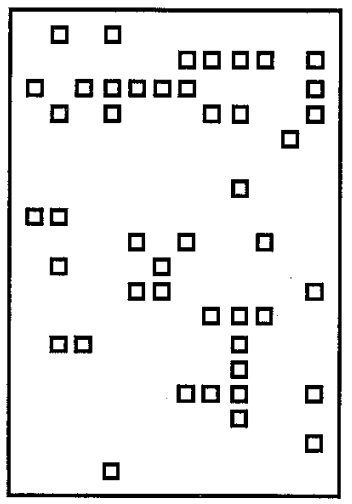
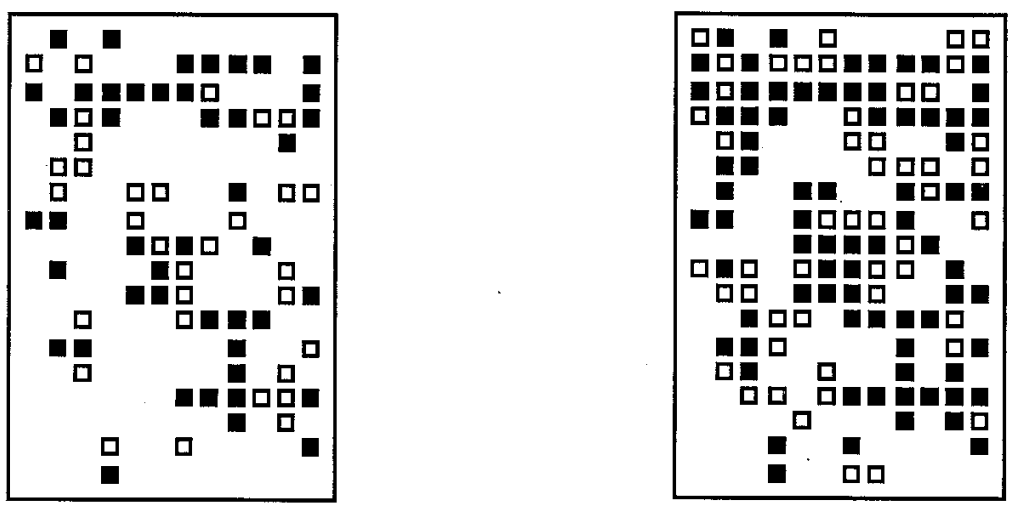

B

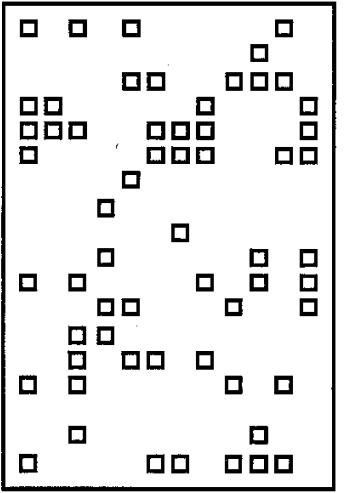
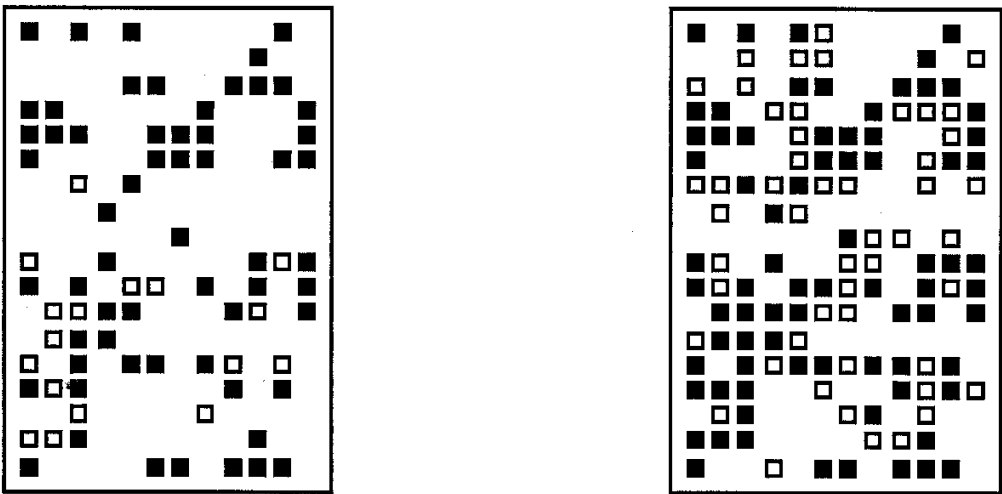

C
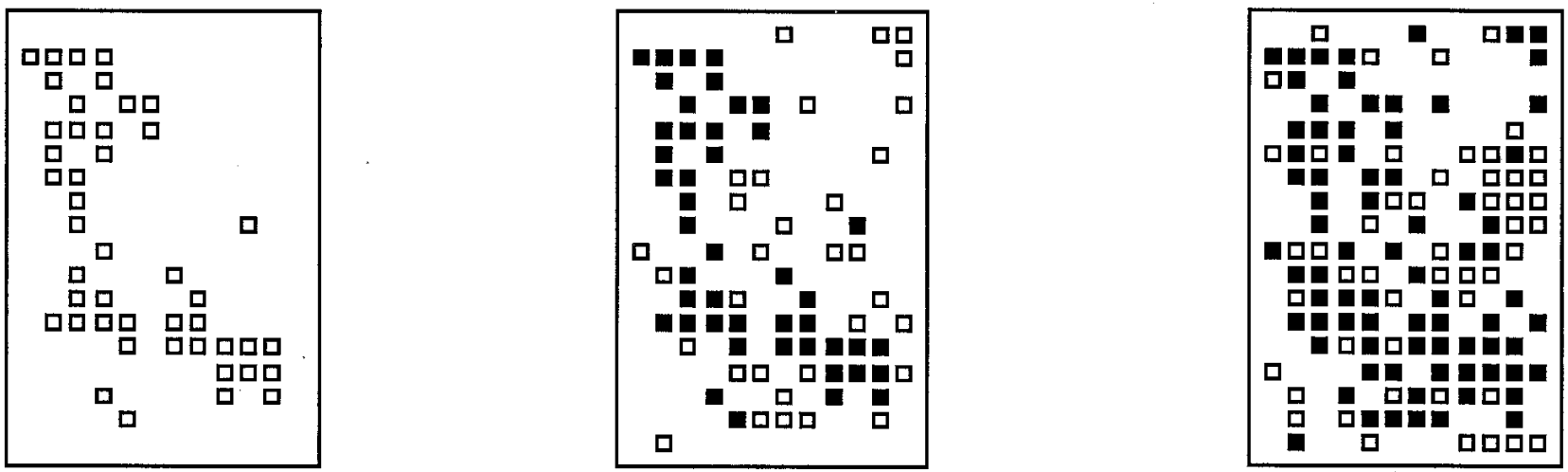

D

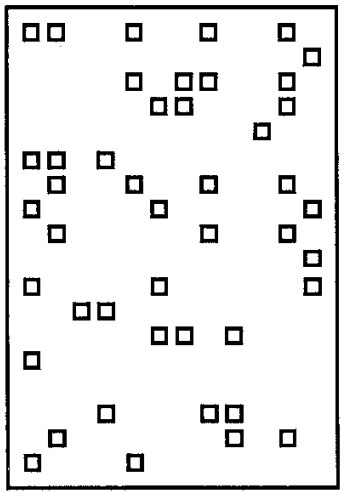
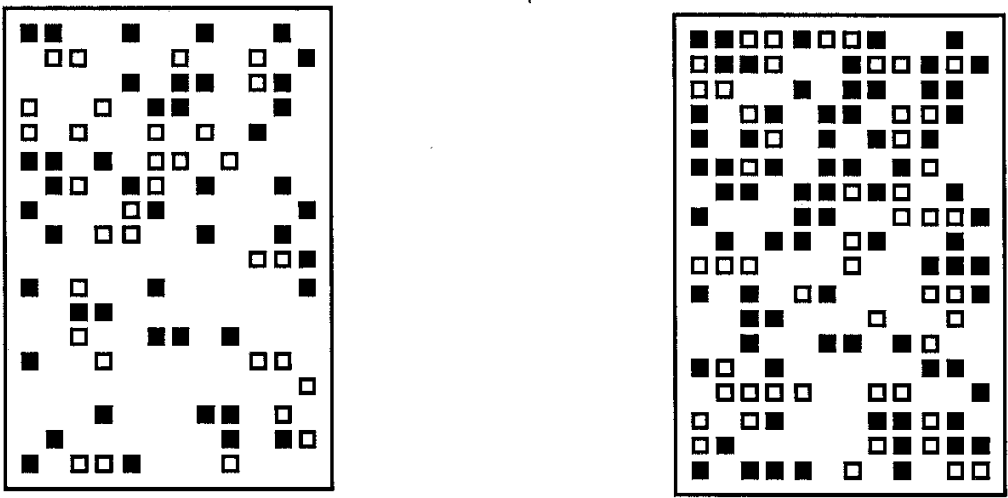

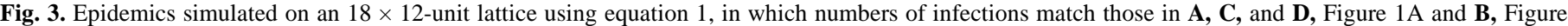
1B. Parameter values used A, $a_{1}=1.15, a_{2}=3.5 ; \mathbf{B}, a_{1}=1.72, a_{2}=1.4 ; \mathbf{C}, a_{1}=0.0, a_{2}=1.0 ;$ and $\mathbf{D}, a_{1}=1.0, a_{2}=0.0$. 
mission effectively 'swamps' the local interactions, while the maximum value of $a_{2}$ corresponds to predominantly nearestneighbor transmission for the local interactions. Thus, the parameter space includes models that approximate the limiting cases discussed in the previous section.

For each simulated epidemic, the following computations were carried out. For each transition $E_{j}, j=1,2,3,500$ samples $\left(a_{i}, \omega_{i}\right)$ were generated from the Markov chain described above (after discarding the initial 20 samples), and the densities $\pi\left(a \mid \omega_{i}, E_{j}\right)$ averaged to provide an estimate of $\pi\left(a \mid E_{j}\right)$. For each simulated epidemic, computation of the three densities required several hours on a workstation, although the computer code has not yet been optimized and improvements in computational efficiency might be made. The product of these densities provided an esti- mate of the density $\pi\left(a \mid E_{1}, E_{2}, E_{3}\right)$, which was then normalized so the maximum value over the lattice was unity. From the lattice of normalized density values, a contour plot over continuous parameter space was estimated using the ContourPlot function in Mathematica (30). The estimates of $\pi\left(a \mid E_{1}, E_{2}, E_{3}\right)$ for the four simulated epidemics are plotted in Figure 4A to D. Approximate confidence intervals for model parameters are estimated from these plots using asymptotic likelihood theory. If $a_{0}$ is the parameter vector that maximizes $\pi\left(a \mid E_{1}, E_{2}, E_{3}\right)$ and $a_{t}$ represents the 'true' model parameter, then the quantity

$$
2\left\{\log \left[\pi\left(a_{0} \mid E_{1}, E_{2}, E_{3}\right)\right]-\log \left[\pi\left(a_{t} \mid E_{1}, E_{2}, E_{3}\right)\right]\right\}
$$

is distributed approximately as $\chi_{d}^{2}$, in which $d$ is the dimension of the parameter space. For the two-parameter model considered
A

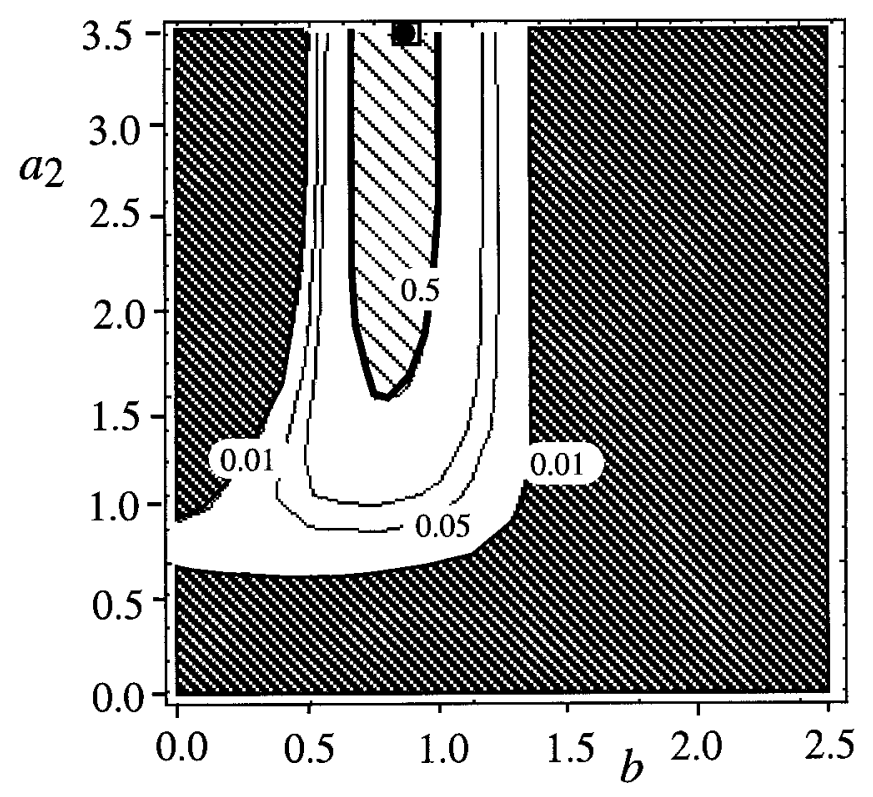

$\mathrm{C}$

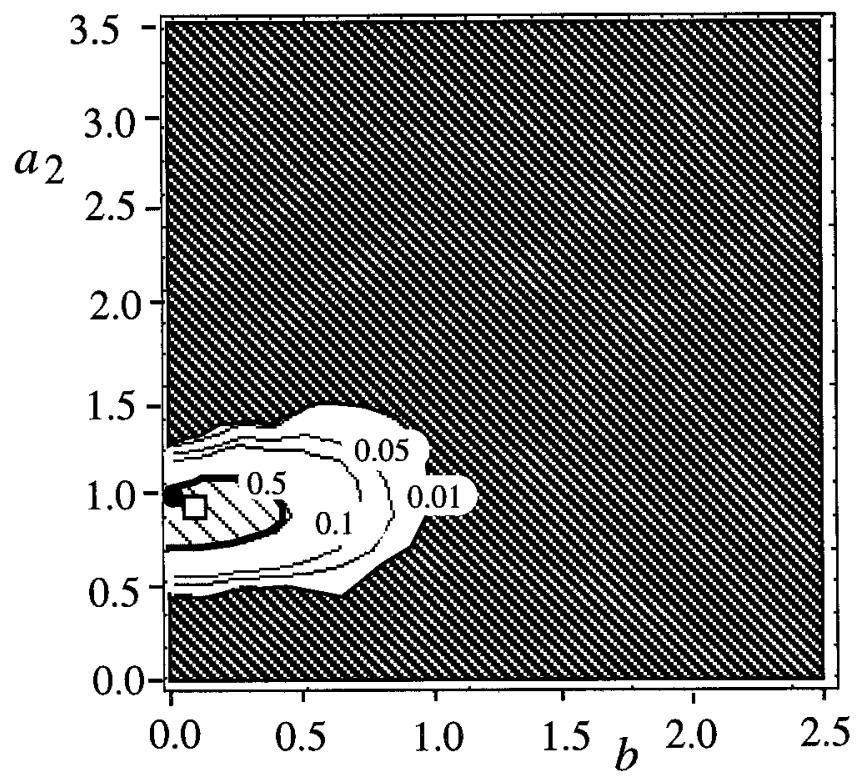

B

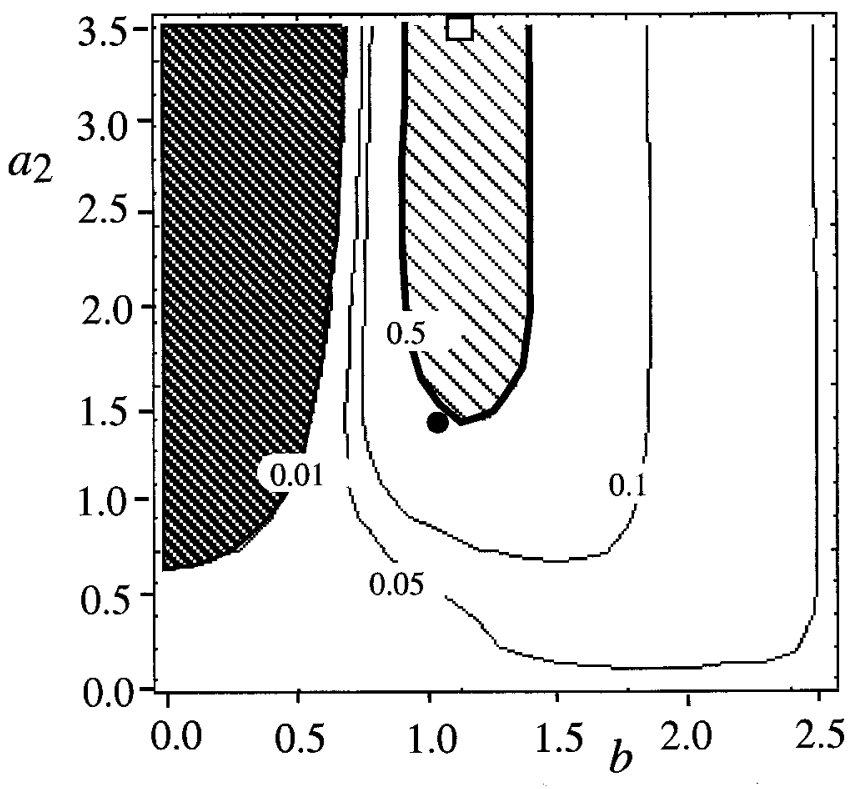

Fig. 4. Contour plots of parameter densities $\pi\left(a \mid E_{1}, E_{2}, E_{3}\right)$, normalized to have maximum value unity, are shown for the epidemics of $\mathbf{A}$ to $\mathbf{D}$, Figures $3 \mathrm{~A}$ to D, respectively. Plots were obtained by estimating value of densities over a $21 \times 21$ grid of parameter values with contours estimated by interpolation using the ContourPlot function in Mathematica (31). Parameter values maximizing density are denoted by a white $\mathbf{A}, \mathbf{B}$, and $\mathbf{C}$, square (or $\mathbf{D}$, rectangle), in which a locus of parameter values maximize density, while parameter values used for the simulations are denoted by a black circle. 
here, it follows that the region of parameter space rejected at significance level $p$ corresponds exactly to the region outside a contour at level $p$ on the normalized density plots. Note that confidence regions may be slightly overestimated by this procedure, since the maximum likelihood parameter vector, $a_{0}$, is unlikely to coincide precisely with a point in the discrete parameter grid or, as is the case with Figure $4 \mathrm{~A}$ and $\mathrm{B}$, may lie outside the range of parameters considered. However, the range of the parameter space, the resolution of the grid, and the transformation applied to $a_{1}$ (equation 3), which prevents the likelihood from fluctuating rapidly over the grid, together mean this issue is not of major concern. Moreover, parameter values rejected at a given significance level using confidence regions estimated here would be rejected a fortiori in the absence of this small bias.

It is apparent that, in each case, the estimated density is consistent with the model parameters used to produce the simulation. For the epidemics of Figure $3 \mathrm{C}$ and D, the corresponding densities in Figure 4C and D strongly support the (accurate) hypotheses that the most parsimonious explanations for the pattern of spread are, respectively, a purely local interaction and a random infection process. The densities in Figure 4A and B illustrate a difficulty that arises in interpreting observations with respect to the model defined by equation 1 . A power-law local transmission has been shown in other studies $(11,25,26)$ to define a model producing spatial patterns exhibiting strong aggregation (suggesting sortrange transmission), while displaying evidence of long-range 'jumps' in the epidemic. Intuitively, this behavior should also be consistent with an epidemic spread by nearest-neighbor transmission, in combination with a random background infection. Although a model with only local transmission would be rejected at the 5\% significance level in both cases, the general shape of the likelihood surfaces of Figure 4A and $\mathrm{B}$ demonstrate that this ambiguity is detected by the method. For other choices of local interaction (e.g., exponentially bounded functions), this would not be so apparent. However, the above ambiguity increases the certainty that a combination of long- and short-range mechanisms are at work when, as with the epidemic of Figure 1A, the analysis suggests that purely local transmission should be rejected.

Application of techniques to CTV epidemics. I now fit the stochastic model defined by equation 1 to the epidemics of Figure 1 using the MCMC technique. For epidemics in both plots, the observations comprise transitions $E_{1}, E_{2}$, and $E_{3}$, in which $E_{1}$ denotes the transition from the disease-free state to the first disease map. The values of $\pi\left(a \mid E_{1}, E_{2}, E_{3}\right)$ were estimated for the two epidemics over the above parameter space. Again, 500 densities were averaged in the estimation of each $\pi\left(a \mid E_{i}\right)$, and 500 mutation cycles were applied between the calculation of each density.

Figure $5 \mathrm{~A}$ and $\mathrm{B}$ show the estimated densities $\pi\left(a \mid E_{1}, E_{2}, E_{3}\right)$ for the epidemics of Figure $1 \mathrm{~A}$ and $\mathrm{B}$, respectively. For the epidemic of Figure 1A, the density plot in Figure 5A strongly indicates that the most likely model incorporates a background infection in combination with a short-range local transmission, $a_{1}=1.15, a_{2}=3.5$, maximizing $\pi\left(a \mid E_{1}, E_{2}, E_{3}\right)$ over the parameter range considered. It casts severe doubts on the notions that the epidemic is a random process or has been spread by purely local interactions.

For the epidemic of Figure $1 \mathrm{~B}$, the parameters that maximize the density of Figure 5B are $a_{1}=1.72, a_{2}=1.575$, and the results are again suggestive of spread resulting from a background infection rate combined with a local interaction. However, the evidence against alternative hypotheses is not so strong as in Figure 5A. While the random pattern is a poor explanation, the notion that the spread is governed by a purely local interaction with a small value of $a_{2}$ (enabling transmissions over large distances) should not be discounted completely. Notice further that the two estimated densities are consistent, with considerable overlap between the $50 \%$ confidence regions.

Limitations of the approach. In common with any stochastic integration technique, the results of MCMC are subject to random errors, and some impression of the size of these can be gained by estimating the standard errors in estimates of $\pi\left(a \mid E_{j}\right), j=1,2,3$, from the 500 densities averaged in the estimates. Typically, for the cases considered in this paper, the (marginal) relative standard errors in the estimates of $\pi\left(a \mid E_{j}\right)$ are around 0.5 to $2.0 \%$ for most values of $a$. However, when $\pi\left(a \mid E_{j}\right)$ is very small, the relative error can be considerably larger (around 10 to $20 \%$ in a very few cases). These relative errors combine additively to produce relative errors in the estimates $\pi\left(a \mid E_{1}, E_{2}, E_{3}\right)$, which may be large for less likely values of $a$. Nevertheless, the estimated density can still be considered a useful indication of the relative likelihood of model parameters in light of the observations, so long as the likelihoodbased confidence intervals that result are not interpreted too literally. Repetition of the estimation procedure using alternative
A

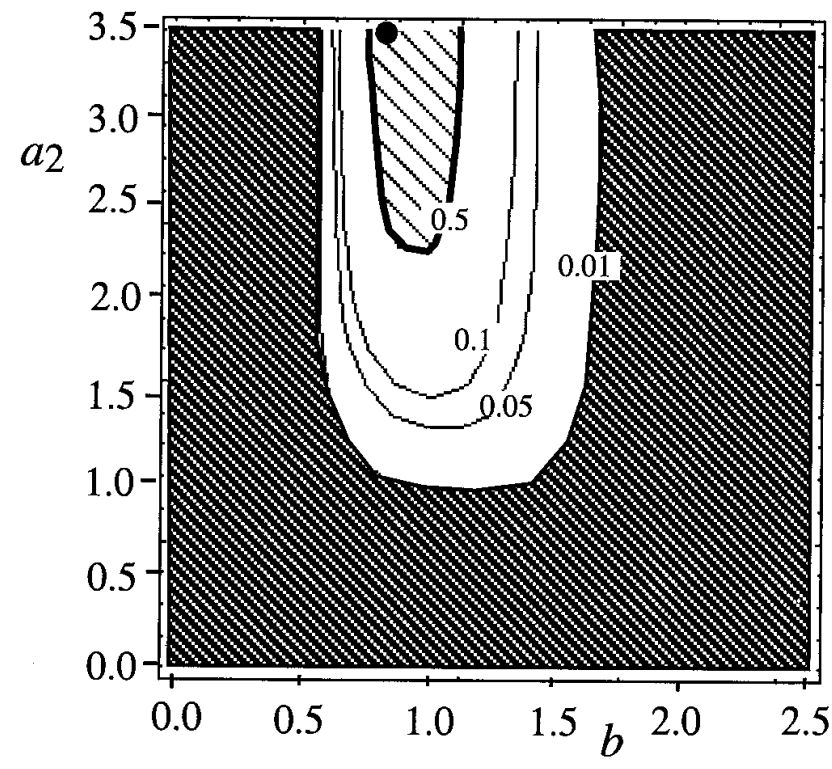

B

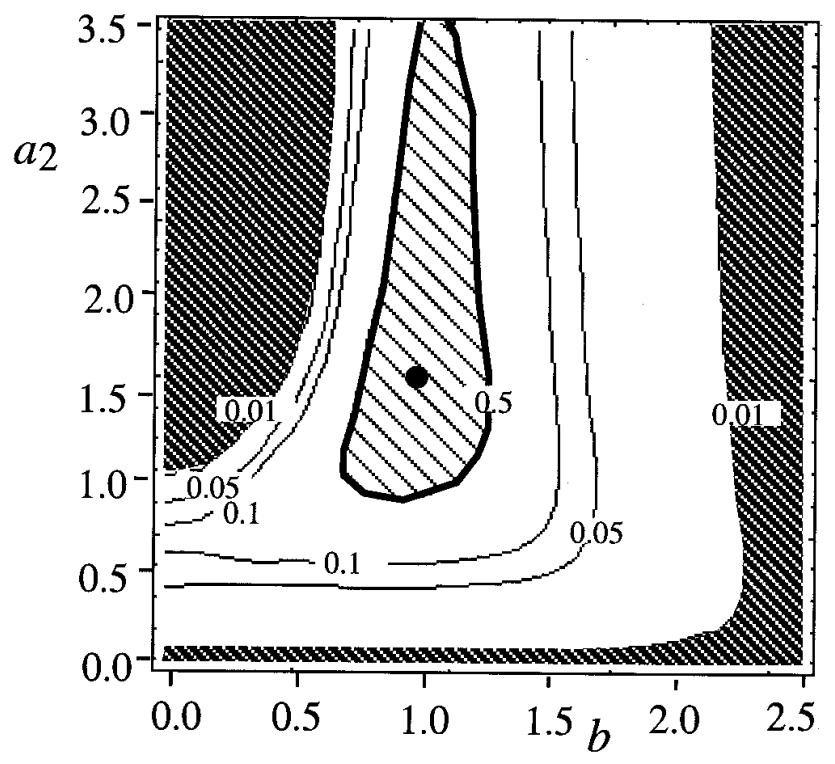

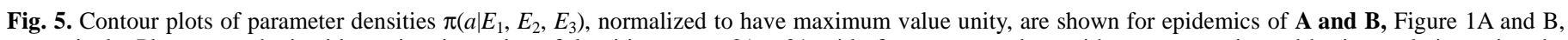

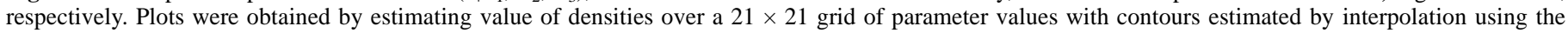
ContourPlot function in Mathematica (31). Parameter values maximizing density are denoted by a black circle. 
random sequences produces largely similar densities. Furthermore, since the relative errors in estimates tend to be smaller for the more likely values of $a$, the method produces consistent maximum likelihood estimates. For very large epidemics, in which many new infections occur between observations, the standard errors in estimated densities become larger, and it may not be possible to estimate densities with both precision and speed using standard computer hardware. In common with many Monte Carlo methods, the techniques of this paper are well suited to implementation on a parallel computer, and this may offer one approach to extending their application to the study of larger systems.

Concluding remarks. Simple stochastic spatiotemporal models are useful in the analysis of intensively mapped observations of spread of viral diseases of plant populations, particularly when sampling is infrequent in time. In this paper, I have illustrated how MCMC methods can be used to fit stochastic models and to translate experimental data directly into conclusions regarding the relative likelihoods of possible mechanisms of transmission. For the case of two epidemics of CTV previously reported and analyzed by Gottwald et al. (12), the results support the idea that both inter- and intraplot transmissions are implicated in the spread of the disease and give some indication of their relative importance. Moreover, they show considerable evidence that local transmission may be predominantly to nearest neighbors in the population, a conclusion that did not emerge from the analyses reported by Gottwald et al. (12). I stress, however, that the analysis presented here ignores other potential factors in the dynamics of the epidemics, such as the presence of edge-effects, which the analyses of Gottwald et al. (12) could detect. Thus, the methods should be seen as complementing other available forms of spatial analysis. Indeed, more standard analyses might be used to investigate questions not addressed by the techniques of this paper. For example, an apparently random transmission mechanism could be the result of random infection from outside a plot ( $a_{1}$ large), intraplot transmission with long-range interactions $\left(a_{2}\right.$ small, $\left.a_{1}=0\right)$, or a combination of these processes. Consideration of nonspatial models for the temporal evolution of incidence levels might shed light on which of these possibilities were most likely.

There is also an important interplay between the spatiotemporal analyses discussed here and purely spatial analyses of pattern (7). As an alternative to Bayesian methods of model selection $(8,14)$, spatial summary statistics can be used as diagnostics for testing the goodness-of-fit of stochastic models. Examples include measures of aggregation or spatial correlation such as join-count statistics (which were used by Gibson and Austin (11) to compare the fit of two models incorporating respectively power-law and exponential local interactions), Geary coefficients, and Moran indices (4). Alternatively, probability distributions can be fitted to aspects of spatial pattern $(16,17)$, and the resulting distributional parameters used as quantitative measures. Thus, it is possible to evaluate the worth of a given stochastic model from its ability to simulate patterns that agree with observation in terms of one or more of the above spatial statistics.

As well as adding to the understanding of the way diseases spread, the stochastic models considered here could also be used in a predictive capacity to compare the likely effectiveness of alternative strategies for the removal and replacement of diseased individuals. The behavior of such models is hard to characterize analytically except for simple cases $(5,6)$. Nevertheless, there is a range of approximation techniques (25) that allow for some mathematical treatment.

As is the case whenever simplistic mathematical models such as equation 1 are used to describe the behavior of a complex biological system, any inferences made must be qualified by the many assumptions implicit in the model. In this paper, the models considered ignore many processes that could have an important bearing on the spatial features of epidemic spread, such as spatial heterogeneity in vector numbers or immunity to infection. Only susceptible and diseased states are considered, and an empirical relationship between infective pressure and distance is assumed. It is also assumed that the characteristics of the transmission process do not change in the period over which observations are gathered. Nevertheless, provided the results are viewed as offering evidence rather than definitive answers regarding mechanisms of epidemic spread, the techniques illustrated in this paper should be useful additions to the range of analytic tools applicable to the analysis of spatiotemporal disease incidence. Moreover, as discussed by Gibson (10), there is no theoretical barrier to extending the methods to more complex models incorporating, for example, removals from the population or to scenarios in which information on a subset of the population may be missing. It is anticipated, therefore, that the approach will be valuable in many situations beyond the scope of this paper.

\section{ACKNOWLEDGMENTS}

This research was funded by the Scottish Office Agriculture Environment and Fisheries Department. I thank C. Glasbey, T. Gottwald, and G. Hughes for helpful comments during the course of this research.

\section{LITERATURE CITED}

1. Bennett, R. J. 1979. Spatial Time Series. Pion Ltd., London.

2. Besag, J. 1977. Discussion of "Spatial contact models for ecological and epidemic spread” by D. Mollison. J. R. Stat. Soc. B 39:315-316.

3. Besag, J., and Green, P. J. 1993. Spatial statistics and Bayesian computation. J. R. Stat. Soc. B 55:25-37.

4. Cliff, A. D., and Ord, J. K. 1981. Spatial Processes: Models and Applications. Pion Ltd., London.

5. Cox, J. T., and Durrett, R. 1981. Some limit theorems for percolation processes with necessary and sufficient conditions. Ann. Probab. 9:583603.

6. Cox, J. T., and Durrett, R. 1988. Limit theorems for the spread of epidemics and forest fires. Stochastic Processes Appl. 30:171-191.

7. Diggle, P. J. 1983. Statistical Analysis of Spatial Point Patterns. Academic Press, London.

8. Draper, D. 1995. Assessment and propagation of model uncertainty (with discussion). J. R. Stat. Soc. B 57:45-97.

9. Gelfand, A. E., and Smith, A. F. M. 1990. Sampling-based approaches to calculating marginal densities. J. Am. Stat. Assoc. 85:398-409.

10. Gibson, G. J. Markov chain Monte Carlo methods for fitting spatiotemporal stochastic models in plant epidemiology. Appl. Stat. In press.

11. Gibson, G. J., and Austin, E. J. 1996. Fitting and testing spatio-temporal stochastic models with applications in plant epidemiology. Plant Pathol. 45:172-184.

12. Gottwald, T. R., Cambra, M., Moreno, P., Camarasa, E., and Piquer, J. 1996. Spatial and temporal analyses of citrus tristeza virus in eastern Spain. Phytopathology 86:45-55.

13. Gottwald, T. R., Reynolds, K. M., Campbell, C. L., and Timmer, L. W. 1992. Spatial and spatiotemporal autocorrelation analysis of citrus canker epidemics in citrus nurseries and groves in Argentina. Phytopathology 82:843-851

14. Green, P. J. 1995. Reversible jump Markov chain Monte Carlo computation and Bayesian model determination. Biometrika 82:711-732.

15. Hastings, W. K. 1970. Monte Carlo sampling methods using Markov chains, and their applications. Biometrika 57:97-109.

16. Hughes, G., and Madden, L. V. 1993. Using the beta-binomial distribution to describe aggregated patterns of disease incidence. Phytopathology 83:759-763.

17. Madden, L. V., and Hughes, G. 1995. Plant disease incidence: Distributions, heterogeneity, and temporal analysis. Annu. Rev. Phytopathol. 33:529-564.

18. Marcus, R., Svetlana, F., Talpaz, H., Salomon, R., and Bar-Joseph, M. 1984. On the spatial distribution of citrus tristeza virus disease. Phytoparasitica 12:45-52.

19. McCullach, P. 1980. Regression models for ordinal data. J. R. Stat. Soc. B 42:109-142.

20. Mollison, D. 1977. Spatial contact models for ecological and epidemic spread (with discussion). J. R. Stat. Soc. B 39:283-326.

21. Mollison, D. 1986. Modelling biological invasions: Chance, explanation, prediction. Philos. Trans. R. Soc. Lond. B 314:675-693.

22. Nelson, S. C. 1995. Spatiotemporal distance class analysis of plant disease epidemics. Phytopathology 85:37-43.

23. Reynolds, K. M., and Madden, L. V. 1988. Analysis of epidemics using 
spatio-temporal autocorrelation. Phytopathology 78:240-246.

24. Reynolds, K. M., and Madden, L. V. 1988. Spatio-temporal analysis of epidemic development of leather rot of strawberry. Phytopathology 78:246-252.

25. Satō, K., Matsuda, H., and Sasaki, A. 1994. Pathogen invasion and host extinction in lattice structured populations. J. Math. Biol. 32:251-268.

26. Shaw, M. W. 1994. Modelling stochastic processes in plant pathology. Annu. Rev. Phytopathol. 32:523-544.

27. Shaw, M. W. 1995. Simulation of population expansion and spatial pat- tern when individual dispersal distributions do not decline exponentially with distance. Proc. R. Soc. Lond. B 259:243-248.

28. Smith, A. F. M., and Roberts, G. O. 1993. Bayesian computation via the Gibbs sampler and related Markov chain Monte Carlo methods. J. R. Stat. Soc. B 55:2-23.

29. Smyth, G. K., Chakraborty, S., Clark, R. G., and Pettitt, A. N. 1992. A stochastic model for anthracnose development in Stylosanthes scabra. Phytopathology 82:1267-1272.

30. Wolfram, S. 1992. Mathematica. Addison-Wesley, Reading, MA. 